\title{
Design and current progress of the Apollon 10 PW project
}

\author{
J.P. Zou ${ }^{1}$, C. Le Blanc ${ }^{1}$, D.N. Papadopoulos ${ }^{1}$, G. Chériaux ${ }^{2}$, P. Georges ${ }^{3}$, G. Mennerat ${ }^{4}$, F. Druon ${ }^{3}$, \\ L. Lecherbourg ${ }^{1}$, A. Pellegrina ${ }^{1,3}$, P. Ramirez ${ }^{1,3}$, F. Giambruno ${ }^{1,2}$, A. Fréneaux ${ }^{1,2}$, F. Leconte ${ }^{1,2}$, \\ D. Badarau ${ }^{1}$, J.M. Boudenne ${ }^{1}$, D. Fournet ${ }^{1}$, T. Valloton ${ }^{1}$, J.L. Paillard ${ }^{1}$, J.L. Veray ${ }^{1}$, M. Pina ${ }^{1}$, P. Monot ${ }^{4}$, \\ J.P. Chambaret ${ }^{4}$, P. Martin ${ }^{4}$, F. Mathieu ${ }^{1}$, P. Audebert ${ }^{1}$, and F. Amiranoff ${ }^{1}$ \\ ${ }^{1}$ Laboratoire pour l'Utilisation des Lasers Intenses, CNRS, Ecole Polytechnique, Palaiseau, France \\ ${ }^{2}$ Laboratoire d'Optique Appliquée, ENSTA ParisTech, CNRS, Palaiseau, France \\ ${ }^{3}$ Laboratoire Charles Fabry, UMR 8501, Institut d'Optique, CNRS, Université Paris Sud, Palaiseau, France \\ ${ }^{4}$ CEA, Iramis, SPAM, Saclay, France \\ (Received 9 July 2014; revised 26 August 2014; accepted 22 October 2014)
}

\begin{abstract}
The objective of the Apollon project is the generation of $10 \mathrm{PW}$ peak power pulses of $15 \mathrm{fs}$ at 1 shot/minute. In this paper the Apollon facility design, the technological challenges and the current progress of the project will be presented.
\end{abstract}

Keywords: titanium lasers; PW class facilities

\section{Introduction}

The main goal of the Apollon project is to provide to users $10 \mathrm{PW}$ peak power pulses at a repetition rate of 1 shot/minute. To reach this extreme peak power level, Apollon will produce extremely short pulses of $15 \mathrm{fs}$ and a corresponding moderate energy of $150 \mathrm{~J}$ after compression. This is in fact the principal difference of Apollon when compared with other multi-PW projects internationally (ELINP, Vulcan 10 PW, SIOM 10 PW project etc.) where pulse duration in the range of 25-40 fs is targeted.

The main 10 PW beam, combined with three secondary beams (a $1 \mathrm{PW}$ beam, a $10 \mathrm{TW}$ probe and the uncompressed high energy beam), will generate intensity on target surpassing the barrier of $2 \times 10^{22} \mathrm{~W} \mathrm{~cm}^{-2}$, capable of producing ultra-intense and ultra-short sources of particles (electrons, protons... ), and coherent and highly energetic $X$ rays. The design and the construction of the Apollon sub-laser systems have been performed in the framework of a French multilaboratory research consortium. Apollon will be integrated as an upcoming user facility at the end of 2014 at l'Orme des Merisiers, Saclay, France.

Correspondence to: J.P. Zou, Laboratoire pour l'Utilisation des Lasers Intenses, CNRS, Ecole Polytechnique, Palaiseau, France.

Email: ji-ping.zou@polytechnique.fr

\section{Apollon architecture strategy}

The Apollon architecture has been defined taking into account the current advances in research and development of the laser technology, laser components and laser materials for ultra-short, ultra-intense laser systems. To produce $10 \mathrm{PW}$ pulses, it is important to determine the output energy level and the ultra-short pulse duration.

Apollon will produce a relatively moderate output energy of $150 \mathrm{~J}$, limited by the size and the damage threshold of the broadband gratings used for pulse compression. In addition, for higher output energy, even larger gain media and more significant pump energy would be required. As a consequence, the transverse lasing risk in such media would be more accentuated and the shot repetition rate compromised.

Apollon will produce high-contrast ultra-short pulses of $15 \mathrm{fs}$, based on excellent spectral bandwidth management and spectral phase minimization all along the chain. In fact, a large spectral bandwidth of about $200 \mathrm{~nm}$ and active spectral phase compensation are needed to support high energy 15 fs pulse generation. However, the main aspects responsible for the pulse duration enlargement and contrast degradation are as follows: the spectral gain narrowing and deformation during energy amplification; the time delay 


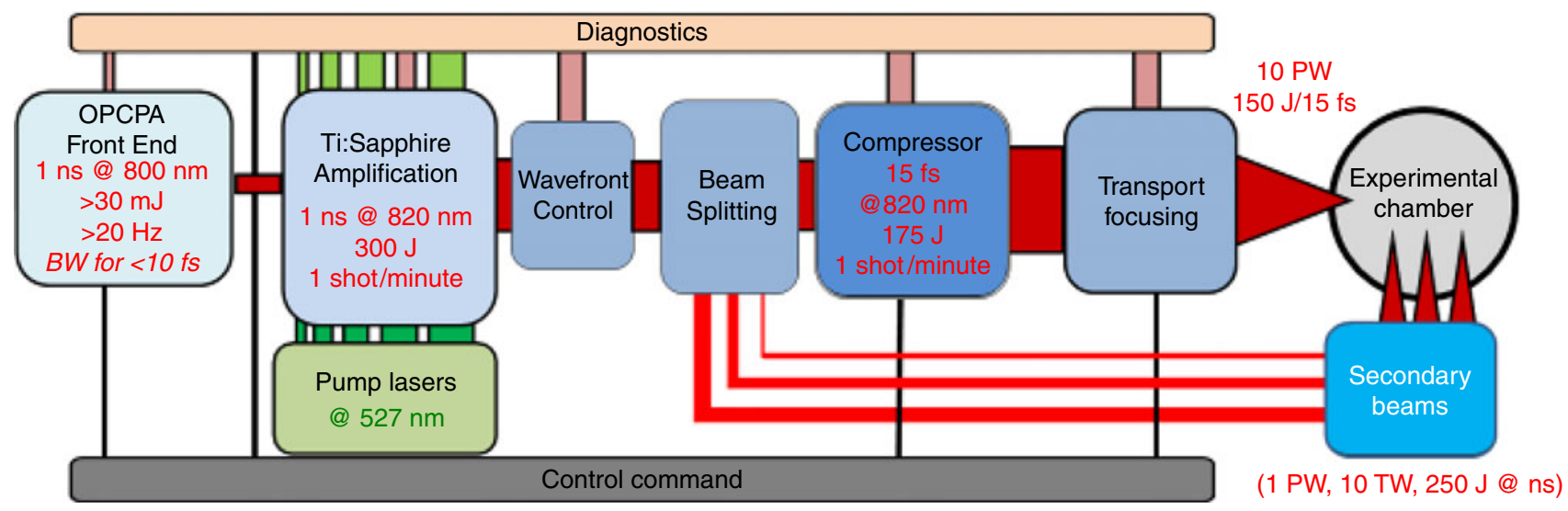

Figure 1. Global schematic of the Apollon $10 \mathrm{PW}$ laser installation.

induced by the longitudinal chromatism generated in spatial filters; the spectral phase induced by material dispersion; and the broadband reflectance limitation and the group delay dispersion (GDD) of the beam-steering mirrors in the chain. For such a laser system, relying on a very broad spectrum, the spatio-temporal coupled effects are of particular importance to study. In the Apollon sub-system design, all of the factors explained in this section will be taken into consideration.

\section{Sub-system design}

To meet the complete set of requirements, the Apollon design is based on a hybrid OPCPA-Ti:sapphire architecture. A simplified schematic of the Apollon laser is shown in Figure 1. The design of the Apollon sub-systems including the front-end, the power amplification section (PAS) and the $10 \mathrm{PW}$ compression will be described in the three following sections.

\subsection{Apollon front-end}

The OPCPA-based Apollon front-end employs state of the art technology to generate high quality and high temporal contrast pulses with a spectrum supporting sub-10 fs pulse duration. A non-collinear OPCPA architecture using BBO crystals has been chosen to allow broad-bandwidth amplification. A particular feature of our design is that the 2-grating aberration-free Offner stretcher is integrated in the front-end between two OPCPA stages. The first OPCPA stage operating in the picosecond regime provides high gain $(1000 \times)$ and temporal contrast enhancement, while the second OPCPA stage operates in a low gain $(50-100 \times)$ but energetic nanosecond regime.

Both the injection pulses at $800 \mathrm{~nm}$ and the pump pulses (SHG of $1030 \mathrm{~nm}$ ) are provided by a broadband Ti:sapphire oscillator (Rainbow, Femtolasers) for optimal temporal synchronization.
The mJ, 25 fs injection pulses (femtopower) are first temporally cleaned and spectrally broadened in a novel double crossed polarized wave (XPW) configuration $^{[1]}$, and then stretched in a bulk stretcher (BK7). The spectral phase is actively controlled by a double passed Dazzler (Fastlite). After a final spatial filtering stage, about $2 \mu \mathrm{J}, 6$ ps stretched pulses with excellent spatial, temporal and spectral quality and stability are injected into the ps-OPCPA stage.

For the OPCPA pump lasers, diode pumped Yb-doped crystal technology is used. The $\mathrm{pJ}$ level pulses at $1030 \mathrm{~nm}$ from the Ti:sapphire oscillator are first temporally stretched and pre-amplified to the $\mathrm{mJ}$ level in the nanosecond regime. Amplification then takes place in two parallel chains: (a) the picosecond line based on an $\mathrm{Yb}: \mathrm{CaF}_{2}$ multipass amplifier providing $>15 \mathrm{~mJ}$ of pump energy at $515 \mathrm{~nm}$ after compression and SHG, and (b) the nanosecond line based on an $\mathrm{Yb}: Y A G$ regenerative amplifier ${ }^{[2]}$ followed by an $\mathrm{Yb}: \mathrm{CaF}_{2}$ booster to reach $>300 \mathrm{~mJ}$ at $515 \mathrm{~nm}$ at $20-100 \mathrm{~Hz}$ repetition rate $^{[3]}$.

So far we have performed OPCPA experiments in the picosecond regime resulting in more than $7 \mathrm{~mJ}$ pulses at $100 \mathrm{~Hz}$. Figure 2 shows an OPCPA output spectral profile and phase (left) and corresponding compressed pulse of 9.5 fs (right) with a contrast better than $10^{-12}$.

\subsection{Apollon power amplification}

The PAS is based on 5 multipass Ti:sapphire amplifiers to obtain $300 \mathrm{~J}$ before compression. Four main points are investigated.

(1) The spectral evolution management, aiming to optimize spectral profile and width compatible with high-contrast 15 fs pulses: spectral filters are designed to fight against gain narrowing and spectral shifting due to the saturation ${ }^{[4]}$. Two sets of these filters will be set up at the entrance of the PAS 

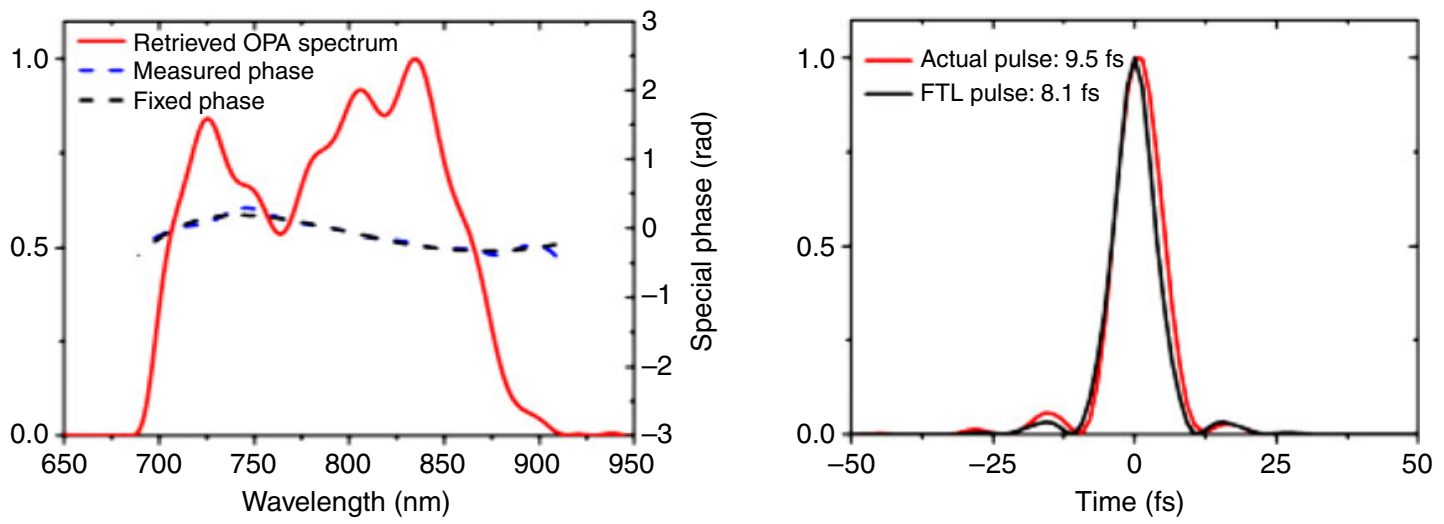

Figure 2. Measurement of the compressed ps-OPCPA pulses: spectral profile and phase (left) and pulse intensity (right).

and before the 3rd amplifier to allow optimal energy amplification while preserving $\sim 70 \mathrm{~nm}$ bandwidth (FWHM) centered at $820 \mathrm{~nm}$. Using another front-end with $50 \mathrm{~nm}$ spectral bandwidth, we have demonstrated spectral management with such filters at the $3 \mathrm{~J}$ level and compressibility down to $23.5 \mathrm{fs}$ of the amplified pulses.

(2) The beam quality control including beam energy distribution optimization and beam wavefront correction. This will be achieved by performing image relay through the whole chain using the well studied off-axis parabolic telescopes and an optical adaptive closedloop. An optimized spatial filtering will also be implemented to minimize the intensity modulations at high spatial frequencies. In this way, the focal spot quality characterized by a Strehl ratio $>0.5$ should be effectively obtained.

(3) Transverse lasing suppression: Apollon employs the largest ever grown crystals, reaching $175 \mathrm{~mm}$ in diameter (GT Crystal Systems) and pumped areas as large as $150 \mathrm{~mm}$ for the last stage. Diameter-to-thickness ratios even greater than 3.5 and high pump energy densities of more than $1 \mathrm{~J} \mathrm{~cm}^{-2}$ make the management of parasitic transverse effects a crucial and challenging task. For the suppression of transverse lasing, novel techniques have been explored based on the use of index-matched liquid. The originality of our approach is related to the online, active control of the minimization of the refractive index difference between the crystal and the liquid. Preliminary experimental tests, in excellent accordance with our simulations, have recently provided solid proof of the efficiency of our method.

(4) High energy pump lasers: a total energy amount of $800 \mathrm{~J}$ in the green is required for pumping the last three stages of the PAS. A commercial pump system developed by Continuum \& National Energetics will

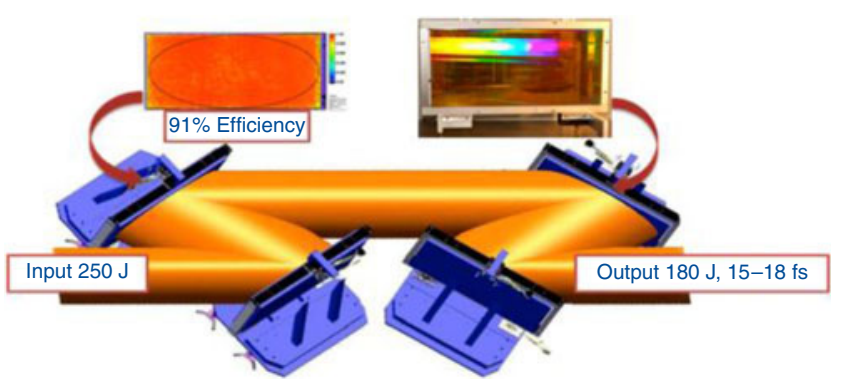

Figure 3. Compressor design with four monolithic gratings. Typical efficiency map for a grating.

be used in Apollon. This laser uses flashlamp pumped large Nd-glass multi-slab amplifiers and liquid cooling allowing an operation rate of at least 1 shot/minute. The first module of the pump system, providing $400 \mathrm{~J}$, will be delivered at the end of 2014 .

\subsection{Apollon pulse compression}

The main beam line compressor of Apollon is based on a typical four grating unfolded configuration (Figure 3). It has been designed on the basis of three principal considerations. The targeted ultra-short pulse duration after compression corresponds to a very large spectral bandwidth $(200 \mathrm{~nm}$ full bandwidth at $820 \mathrm{~nm}$ ) and needs accurate spectral phase management. The damage threshold of the gratings is also an important issue. In fact, to deliver $10 \mathrm{PW}$ pulses to the experiments, energy of $180 \mathrm{~J}$ is required at the output of the compressor, corresponding to about $250 \mathrm{~J}$ at the input for a compression assuming an efficiency of $>70 \%$. Existing grating technology, however, limits our choice to gold-coated gratings as the only compatible solution with the required bandwidth of 720-920 $\mathrm{nm}$ and a damage threshold in the range of $200-300 \mathrm{~mJ} \mathrm{~cm}^{-2}$. In our design, the peak fluence on the first grating of $<110 \mathrm{~mJ} \mathrm{~cm}^{-2}$ imposes the use of a $400 \mathrm{~mm}$ beam size and therefore meter-long gratings. 
The Apollon compressor employs gratings of $910 \times$ $455 \mathrm{~mm}^{2}$ manufactured by Laurence Livermore National Laboratory (LLNL). Since January 2012, six of these gratings have been received and fully characterized. The gratings have $1480 \mathrm{l} \mathrm{mm}^{-1}$ and their diffraction efficiency has been measured to be better than $92 \%$ on three wavelengths ( 780 , 800 , and $840 \mathrm{~nm}$ ). The wavefront error of the diffracted beam is lower than $\lambda / 3 \mathrm{PtV}$ over the whole effective surface. Taking into account the geometrical limitations due to the large beam section, the incident angle has been set at $56^{\circ}$. The spectral phase induced by the material dispersion in the laser chain, as well as the GDD of the mirror coating, has then been minimized based on the design of an unmatched stretcher in the front-end which uses two $14501 \mathrm{~mm}^{-1}$ gratings at the incident angle of $52.4^{\circ}$. The compensation of the residual higher order spectral phase by Dazzler will enable us to reach a final duration below $15 \mathrm{fs}$ after pulse compression.

The compressor will be implemented in a large stainless steel vacuum chamber $\left(6.2 \times 3 \times 3.1 \mathrm{~m}^{3}\right)$ specified to provide a vacuum level of $10^{-7} \mathrm{mbar}$ and cleanliness of ISO6. The large size of the chamber allows entry to the compressor from a single entrance and handling and alignment of the gratings inside the chamber. Delivery of the chamber is scheduled for September 2014.

\section{Acknowledgements}

The authors gratefully acknowledge financial support from the ILE-APOLLON 07-CPER 017-01 contract.

\section{References}

1. L. P. Ramirez, D. N. Papadopoulos, A. Pellegrina, P. Georges, F. Druon, P. Monot, A. Ricci, A. Jullien, X. Chen, J. P. Rousseau, and R. Lopez-Martens, Opt. Express 19, 93 (2011).

2. J. Tümmler, R. Jung, H. Stiel, P. V. Nickles, and W. Sandner, Opt. Lett. 34, 1378 (2009).

3. D. N. Papadopoulos, F. Friebel, A. Pellegrina, M. Hanna, P. Camy, J.-L. Doualan, R. Moncorge, P. Georges, and F. Druon, IEEE JSTQE 21, 1 (2015).

4. F. Giambruno, C. Radier, G. Rey, and G. Chériaux, Appl. Opt. 50, 2617 (2011). 